\title{
Exciton states in GaAs/AlGaAs Bragg confining structures studied by resonant Raman scattering
}

\author{
M. ZAHLER, E. COHEN, J. SALZMAN, E. LINDER and L.N. PFEIFFER* \\ Solid State Institute, Technion- Israel Institute of Technology, Haifa 32000, Israel \\ * AT\&T Bell Laboratories, Murray Hill, NJ 07974, U.S.A.
}

\begin{abstract}
We report on the study of the low temperature resonant Raman scattering (RRS) in $\mathrm{GaAs} / \mathrm{Al}_{\mathbf{0 . 3 2}} \mathrm{Ga}_{0.68} \mathrm{As}$ Bragg confining structures (BCS). The LO-phonon $R R S$ is observed in the spectral range of the $\left(e_{B}: h h_{B}\right)$ and $\left(e_{B}: l h_{B}\right)$ Bragg confined excitons. The RRS profiles show an outgoing beam resonance that is much stronger than the incoming beam resonance. These profiles are analyzed by a model in which the exciton dimensionality affects the in-plane excitonic dispersion and the Fröhlich interaction dependence on the phonon wavevector. The exciton scattering, in its translational motion, is assumed to be due to heavy point defects (short range potential fluctuations). Detailed calculations of the RRS profiles result in excellent agreement with the observed spectra. From them we conclude that the Bragg confined $\left(e_{B}: h h_{B}\right)$ exciton is approximately 2-dimensional.
\end{abstract}

\section{Introduction}

In Bragg confining structures (BCS), the electron and hole are confined (in their motion perpendicular to the quantum layers) by Bragg reflections from superlattice (SL) sections [1,2]. This results in a discrete energy level (denoted $e_{B}, h h_{B}$ and $l h_{B}$ for the electron, heavy and light hole, respectively) that is higher than those of the SL sections. Fig.1 describes the GaAs/AlGaAs BCS's studied here and the carrier wavefunctions along the confinement direction. Bragg confined electron and exciton states have been experimentally observed $[3,4]$ by various methods. Here we report on a study of the LO-phonon resonant Raman scattering (RRS) that is mediated by the Bragg confined excitons. The observed RRS profiles (scattering intensity as a function of excitation (laser) energy, $E_{L}$ ) show a strong asymmetry between the incoming beam and outgoing beam resonances. These profiles are analyzed by a model based on the exciton translational motion (in the presence of random potential fluctuations) and on the Fröhlich interaction between the exciton and the LO-phonons. It is shown that exciton dimensionality affects the RRS profile, and from the analysis we deduce that it is approximately 2 -dimensional.

\section{Experiment}

All the structures studied here were grown by molecular beam epitaxy on (001) - oriented GaAs substrates. The BCS's consist of SL sections, each one has 5 periods of 8 monolayers (ml's) of GaAs wells and $22 \mathrm{ml}$ 's of $\mathrm{Al}_{0.32} \mathrm{Ga}_{0.68} \mathrm{As}$ barriers. Between these SL sections there are $\mathrm{Al}_{0.32} \mathrm{Ga}_{0.68} \mathrm{As}$ spac- 
ers, either 66 ml's thick (A) or 44 ml's thick (B). The "unit cell" of the BCS is schematically shown in Fig. 1, by the band gap variation of the constituent materials along the growth direction. The band offset ratio is taken as $V_{c}: V_{v}=6: 4$. Also shown are the calculated $e$ and $h$ subbands in the Bragg confined energy levels $\left(e_{B}\right.$ and $\left.h h_{B}\right)[3]$. The unit cell is repeated 60 times. All samples are undoped and were grown without interruptions at the interfaces. For the spectroscopic measurements, the samples were placed in an immersion type dewar (at $\mathrm{T}=2 \mathrm{~K}$ ) and excited by $\mathrm{cw}$ dye lasers pumped with an $\mathrm{Ar}^{+}$laser. The dye laser linewidth was $0.1 \mathrm{meV}$ and the intensity at the sample surface was varied in the range of $0.1-20 \mathrm{~W} / \mathrm{cm}^{2}$. A backscattering geometry was used, and the scattered radiation was dispersed by a double monochromator (resolution of $0.05 \mathrm{meV}$ ).

Fig.2 shows the photoluminescence (PL) and its excitation (PLE) spectra of the two BCS's studied here. The RRS consists of GaAs-like and AlAs-like LO phonon bands. The integrated intensity of the GaAs-like LO-phonon is shown by circles in Figs.2c and 2f. These are the experimental RRS profiles. Similar ones are obtained for the AlAs-like LO-phonon.

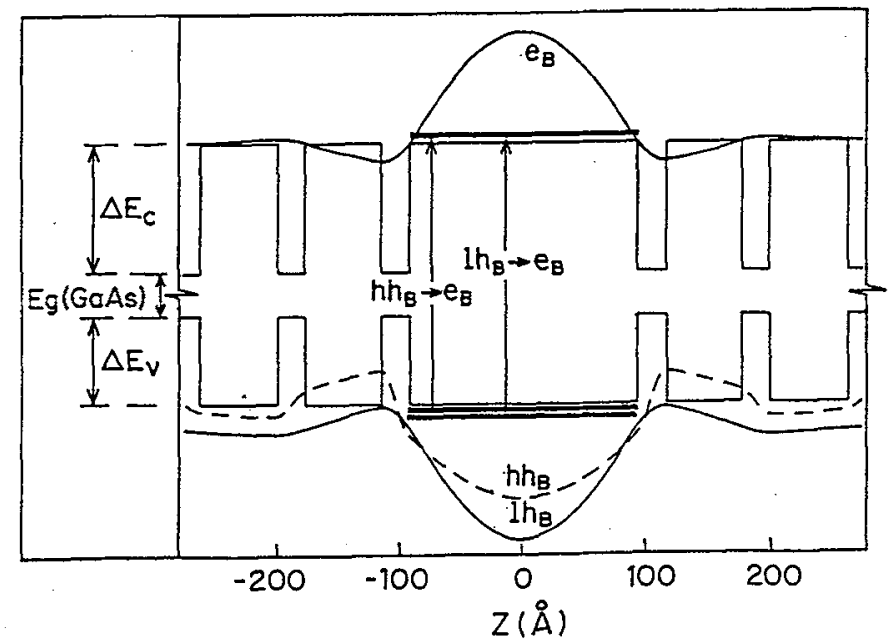

Fig.1: The one dimensional "unit cell" of the Bragg confining structures represented by the conduction and valence bands. The band offset ratio is 6:4. Note the Bragg confined states $e_{B}$ and $h h_{B}$ in the spacers, and their wavefunctions. 


\section{Discussion}

The two bands observed either in PL or in PLE (Figs.2a-d) are due to the radiative recombination of the $\left(e_{B}: h h_{B}\right)$ and $\left(e_{B}: l h_{B}\right)$ Bragg confined excitons. Their intensity is much weaker than that of the (el: $h h 1$ ) excitons, in the SL sections (at $1.754 \mathrm{eV}$, not shown in Fig.2). In spite of that, there is a very strong $R R S$ in the spectral range of the Bragg confined excitons. We note that in the BCS A, the RRS peaks are observed at the $\left(e_{B}: h h_{B}\right)$ exciton and at $\hbar w_{L O}$ above it. (These are the incoming beam (IB) and outgoing beam (OB) resonances, respectively). In the case of BCS $B$, the RRS IB and OB are closer to the $\left(e_{B}: l h_{B}\right)$ than to the $\left(e_{B}: h h_{B}\right)$ exciton. We calculated the degree of exciton confinement in the spacer layers for the two BCS. For the BCS A, the $\left(e_{B}: h h_{B}\right)$ exciton has a higher confinement factor than the $\left(e_{B}: l h_{B}\right)$ exciton, while for BCS B this relation is reversed. We thus propose that exciton damping, which controls the RRS intensity depends on the degree of confinement.

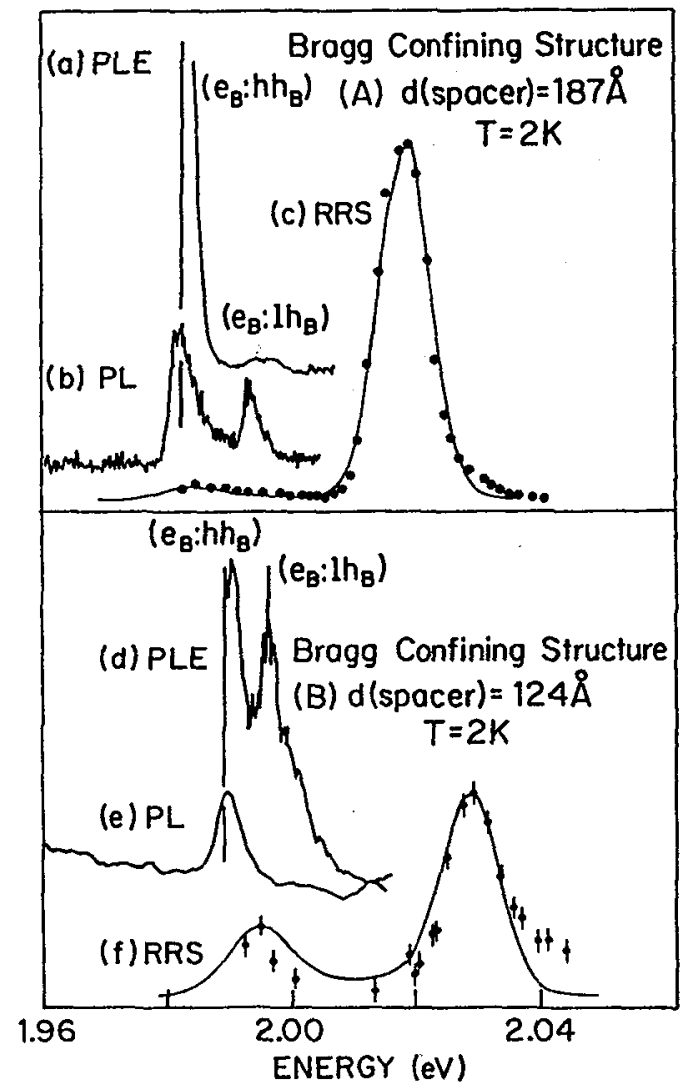

Fig.2: (a) The photoluminescence excitation spectrum of the BCS A monitored at the peak of the $\left(e_{B}: h h_{B}\right)$ exciton band. (b) The photoluminescence spectrum excited at $2.41 \mathrm{eV}$. (c) The resonant Raman scattering profile. Circules are the observed intensities of the GaAs-like LO-phonon scattering. The solid curve is the model calculation. (d), (e), (f) are the respective spectra of the BCS B. 
In order to calculate the RRS profiles we use a model based on exciton scattering by random potential fluctuations in the Bragg confining spacer layer [5]. The potential fluctuations are mainly due to alloy composition fluctuations and are assumed to be uncharged point defects that act only on the in-plane exciton motion. Consequently, exciton states with $\vec{K}_{\|}=0$ and $\vec{K}_{\|} \neq 0$ are admixed, and this gives rise to a double resonance in the case of the $O B$ scattering $[6,7]$. Details of this model are published elsewhere [5], and here we present only the calculated RRS profiles (solid curves in Figs.2c and 2f). The most important conclusion that can be drawn from the good fit of this model to the experimental RRS profiles concerns the dimension of the $\left(e_{B}: h h_{B}\right)$ and $\left(e_{B}: l h_{B}\right)$ excitons. The exciton wavefunction (in the $1 \mathrm{~S}$ state) is anisotropic with regard to the confinement direction and the layer plane. This is represented by Bohr radii $a_{z}$ and $a_{\|}$, respectively. The ratio of the fitted parameters, $a_{\| \mid} / a_{z} \sim 10$, indicates that the exciton in the Bragg confining layers is approximately 2-dimensional.

Acknowledgments: The work at Technion was supported by the United States-Israel Binational Science Foundation (BSF), Jerusalem, Israel and was done at the Center for Advanced OptoElectronics Research.

\section{References}

1. F.H. Stillinger, Physica. B85, 270-276 (1977).

2. G. Lenz and J. Salzman, Appl. Phys. Lett. 56, 871 (1990).

3. M. Zahler, I. Brener, G. Lenz, J. Salzman and E. Cohen, Appl. Phys. Lett. 61, 949 (1992).

4. C. Sirtori, F. Cappaso, J. Faist, D.L. Sivco, S-n.G. Chu and A.Y. Cho, Appl. Phys. Lett. 61, 898 (1992).

5. M. Zahler, E. Cohen, J. Salzman E. Linder and L.N. Pfeiffer, Phys. Rev. Lett. 71, (1993).

6. I. Brener, E. Cohen, A. Muranevich and R. Triboulet, Phys. Rev. B40, 8313 (1989).

7. A.J. Shields, M. Cardona, R. Nötzel and K. Ploog, Phys. Rev. B46, 10490 (1992). 